\title{
Análisis de la contribución del gasto público de seguridad en la mitigación de la delincuencia de las entidades federativas de México
}

\author{
Analysis of the Contribution of Public Spending on Security in Mitigating \\ Crime in the States of Mexico \\ Carlos Heriberto Flores Orona, Rafael Eduardo Saavedra Leyva \\ y Gilberto Martínez Sidón*
}

Resumen: El presente artículo tiene por objetivo analizar la contribución del gasto público destinado a seguridad en la mitigación de la delincuencia en las entidades federativas de México. Desde la teoría empírica queda el debate sobre si efectivamente el mayor gasto en seguridad coadyuva en la disminución de la delincuencia. Para cumplir el objetivo se emplea un micro panel balanceado. Se utilizan múltiples variables de delincuencia para contrastar los resultados y robustecer las conclusiones. Además, se utilizan el salario, la educación y el desempleo como variables de control y explicativos de la delincuencia. Los resultados sugieren que existe una relación negativa y estadísticamente significativa entre el gasto público destinado a seguridad y la disminución de la actividad delictiva. Asimismo, un mayor salario coadyuva en la mitigación de los delitos, delitos contra el patrimonio y delitos contra la libertad personal.

Palabras clave: seguridad, delincuencia, delitos, gasto público, México.

Abstract: The objective of this article is to analyze the contribution that public spending for security has in mitigating crime in the states of Mexico. From the empirical theory, the debate remains as to whether the higher spending on security actually contributes to the decrease in crime. To reach the objective, a balanced micro panel is used. Multiple crime variables are

\footnotetext{
*Carlos Heriberto Flores Orona es profesor de cátedra en el Instituto Tecnológico y de Estudios Superiores de Monterrey (ITESM) campus Chihuahua, Calle privada de Corregidora, 3101, colonia Santo Niño, Chihuahua, Chihuahua. Tel: 614495 6370. Correo-e: chflores@tec.mx. ORcid: https://orcid.org/0000-00016304-9643. Rafael Eduardo Saavedra Leyva es profesor de tiempo completo de la Facultad de Ciencias Químicas e Ingenierías, Universidad Autónoma de Baja California, Calzada Universidad, 14418, Parque Industrial Internacional, Tijuana, 22390, México. Tel: 667255 1021. Correo-e: eduardo.saavedra@uabc. edu.mx. ORCiD: https://orcid.org/0000-0001-9200-4997. Gilberto Martínez Sidón es profesor-investigador del Departamento Académico de Economía, Universidad Autónoma de Baja California Sur, Cuauhtémoc, 675, entre Altamirano y Gómez Farías, colonia Pueblo Nuevo, La Paz, Baja California Sur, México, 23097. Tel: 664651 0157. Correo-e: gmartinez@uabcs.mx. orciD: https://orcid.org/0000-0001-7642-6321.

Artículo recibido el 8 de noviembre de 2020 y aceptado para su publicación el 2 de agosto de 2021.
} 
used to contrast the results and strengthen the conclusions. Likewise, salary, education and unemployment are used as control variables and explanatory of crime. The results suggest that there is a negative and statistically significant relationship between public spending on security and the decrease in criminal activity. Likewise, a higher salary contributes to the mitigation of crimes, crimes against property and crimes against personal freedom.

Keywords: security, delinquency, crime, public spending, Mexico.

\section{INTRODUCCIÓN}

e reconoce que la inseguridad es un factor que afecta no solo a la actividad $\checkmark$ económica, sino a todas las actividades sociales. Garantizar la seguridad pública es un reto que enfrentan los gobiernos. En este sentido, un parámetro para establecer la consolidación de la seguridad pública es a través del aumento o disminución de la delincuencia; por ello, desde la administración pública se establecen diversas estrategias para garantizar la seguridad pública, mismas que van desde modificaciones a la ley, hasta el incremento de recursos monetarios destinados a la seguridad. Dado que son múltiples los factores que inciden en la actividad delictiva, y cada uno de ellos amerita un estudio detallado, el presente artículo centra el análisis en el gasto público ejercido en seguridad como mecanismo para mitigar la actividad delictiva.

En la diversa literatura sobre el gasto público en seguridad queda el debate sobre el impacto que tiene el gasto destinado a la seguridad en la disminución de la actividad delictiva. Sirva de ejemplo el estudio de Foglia (2017), en donde reconoce que el gasto público en seguridad en muchos casos no se ha traducido en una disminución de la delincuencia. De hecho, argumenta que a pesar de que los países tengan tasas de crecimiento positivas, el nivel de inseguridad se ha incrementado. Por esto, más que aumentar el gasto público en seguridad, lo que se recomienda es encontrar otros mecanismos para la mitigación de la actividad delictiva o una mejor asignación del recurso.

En este sentido, Jaitman y Keefer (2017) sostienen que la mayor parte del gasto público en seguridad se asigna a las policías y, en menor medida, el gasto se asigna a la aplicación de justicia y la prevención. Por lo anterior, se reconoce la necesidad de desarrollar políticas de prevención de delitos y políticas de gasto en seguridad de largo plazo, que busquen atacar las causas y no las consecuencias de la delincuencia. De acuerdo con Soria (2018), si el gasto en seguridad pública se orienta a prevenir la inseguridad, gasto social, infraestructura u otro rubro que repercuta en el cambio cultural, el crecimiento económico y la generación de empleo permitirían impulsar una mayor igualdad social y de oportunidades, lo que se traduciría en una disminución de la delincuencia. 
$\mathrm{Al}$ respecto, Sumano (2020) argumenta que la eficacia de la política de prevención de la delincuencia dependerá de las instituciones y controles sociales que estén presentes en la sociedad. Por ejemplo, el autor señala que un programa de prevención de violencia escolar no será exitoso si no se combina con programas que fomenten estilos de crianza positiva. Los programas para la crianza positiva no serán exitosos para los jóvenes sin la presencia de oportunidades. Los programas de inserción laboral para los jóvenes no serán exitosos si no se complementan con calles vigiladas por la policía. Por lo tanto, la prevención social de la delincuencia sólo podrá tener éxito cuando exista un contexto institucional suficientemente fuerte en la comunidad que la sustente.

En este sentido, Zúñiga (2016) destaca que la delincuencia es perjudicial en distintos ámbitos de la economía y la sociedad, pues constituye una amenaza específica para la supervivencia de las personas honestas, de negocios y la seguridad de los consumidores. Así, no solo es suficiente la certeza en el comportamiento de los mercados lo que productores como consumidores necesitan, sino que la seguridad pública es un factor que proporciona confianza para el desarrollo positivo de la economía y la sociedad.

Asimismo, Cortez y Grijalva (2021) establecen que, desde el punto de vista de los incentivos económicos, la explicación de la delincuencia ha sido considerada desde dos perspectivas. Por un lado, está la visión individual, la cual se centra en la decisión de las personas de cometer o no un delito y las variables que influyen en su resolución. Por otro lado, está la visión agregada, en la que se considera que las diferencias que existen en la incidencia criminal en un nivel agregado se deben a la influencia estructural de factores sociales y económicos del entorno.

Por lo tanto, y más allá de la asignación del gasto público en seguridad en los diferentes rubros, el debate también se ha centrado en la cantidad de recurso monetario destinado al tema de seguridad. Por ejemplo, en los países de América Latina, donde la delincuencia ocupa el primer lugar entre los problemas más importantes, de acuerdo con la opinión pública, incluso por encima de la corrupción y otros problemas económicos, el gasto en seguridad pública representa en promedio 5 por ciento del gasto público total, lo que equivale a una tercera parte de lo que se gasta en educación y salud. Esto va de la mano de una fuerte demanda por parte de la sociedad de tener mejores niveles de vida y seguridad. En este contexto, el presupuesto destinado a la seguridad pública parece insuficiente (Foglia, 2017).

De acuerdo con la Encuesta de Seguridad Urbana (Ensu), el principal problema social que enfrentan los mexicanos son la inseguridad y la delincuencia (Soria, 
2018); estas mismas afirmaciones también son señaladas por la Encuesta Nacional de Calidad e Impacto Gubernamental (ENCIG). En esta última, desde 2011 hasta 2017, en promedio, 70 por ciento de las personas coincide en que el tema de inseguridad y delincuencia es lo que más afecta a la sociedad en México, y un aspecto que agrava esta situación es la corrupción; por ello, subsanar el problema de inseguridad ayudaría a mejorar los niveles de la actividad económica y de bienestar social (Martínez y Morones, 2019).

Así pues, en México, de acuerdo con el Centro de Investigación Económica y Presupuestaria (2019), el gasto en seguridad pública de 2008 a 2019 representó en promedio alrededor del uno por ciento del producto interno bruto (PIB). Para 2019, el gasto en seguridad representó apenas 0.96 por ciento del PIB, 14.3 por ciento menos que en $2018 ; 0.96$ por ciento representa 4 por ciento del gasto total aprobado. El destino del gasto en seguridad pública se divide en tres ejes:

1. Prevención del delito, que representa 14 por ciento del gasto. Involucra:

a) Prevención, protección y fortalecimiento de seguridad pública.

b) Acciones del gobierno para el respeto a derechos.

c) Promoción y prevención de derechos humanos.

d) Acceso a la información.

2. Combate al crimen, que representa 42 por ciento. Involucra:

a) Coordinación de la seguridad pública.

b) Combate a la corrupción.

c) Fuerzas armadas en apoyo a la seguridad pública.

3. Justicia, que representa 44 por ciento del gasto. Involucra:

a) Investigar y perseguir.

b) Impartición de justicia, reclusión y readaptación social.

c) Atención, observación, defensa y supervisión de los derechos humanos.

d) Asesoría jurídica.

Al respecto, Fuentes y Sánchez (2017) argumentan que las acciones que se han llevado a cabo en México para combatir la delincuencia se han centrado en medidas reactivas y poco se hace en términos de generar acciones preventivas que consideren un contexto sociodemográfico en donde se presentan las oportunidades para que se cometan los delitos.

De acuerdo con datos de Instituto Nacional de Estadística y Geografía (INEGI, 2019), la Encuesta Nacional de Victimización y Percepción sobre Seguridad Pública (Envipe) señala que 33.9 por ciento de los hogares en México contó con por 
lo menos un integrante, víctima del delito. En 93.2 por ciento de los delitos no hubo denuncia, o no hubo inicio de una averiguación previa o carpeta de investigación. Asimismo, los datos señalan que de 2017 a 2018 hubo una disminución en la tasa de incidencia delictiva, que pasó de 39369 delitos por cada cien mil habitantes en 2017 a 37807 delitos por cada cien mil habitantes en 2018.

En este contexto, donde el tema de la inseguridad tiene especial interés en la agenda económica y social, el presente documento tiene por objetivo analizar y cuantificar la contribución que tiene el gasto público destinado a seguridad pública en la mitigación de la delincuencia en las entidades federativas de México. En adelante el documento se estructura como sigue: en el segundo apartado, "Marco de discusión", se presenta la discusión teórica y empírica; el tercer apartado, "Metodología", la metodología y el análisis de estadística descriptiva; en el cuarto apartado se presentan los resultados, y en el último se presentan las conclusiones.

\section{MARCO DE DISCUSIÓN}

El análisis empírico de la actividad delictiva en la economía se remonta, según Becker (1968), en la ponderación del costo de oportunidad de la mejor situación que le atrae a un individuo la relación entre beneficio o castigo. En esta toma de decisión casi espontánea; algunos individuos toman la decisión de llevar a cabo alguna actividad delictiva. Dicha actividad se convierte en un problema de inseguridad que afecta no solo a la actividad económica sino también a la sociedad en su conjunto. Por ello, los gobiernos formulan y emplean diversas estrategias para mitigar el problema de la inseguridad, y un mecanismo para hacerlo es a partir del gasto público.

Por otro lado, Vilalta y Fondevila (2018) mencionan que la prevención del delito no es materia de experimentación científica, pero sí requiere un acervo de conocimiento sobre las buenas y las malas prácticas. En todo caso, en el contexto presente, lo que se debe buscar rápida y colaborativamente es una reducción del daño económico y de los efectos negativos que tiene en la sociedad un empresariado asediado por el delito.

De esta manera, a nivel social, dadas las altas tasas de delincuencia, el gobierno se ve obligado a gastar una parte significativa de su presupuesto en prevención del delito, en la financiación de agencias encargadas de aplicar la ley, en gestión de centros de detención y cárceles, y en la implementación de programas de seguridad pública, por lo que un mayor gasto público en seguridad resultaría razonable (Daniele y Marani, 2008). 
No obstante, en algunos casos este mecanismo de solo aumentar el gasto público en seguridad no ha sido suficiente para resolver el problema, tal es el caso de Grecia, donde el gasto en seguridad no produjo una reducción de la actividad delictiva. Este resultado se explica por políticas anticrimen fallidas y vigilancia policial improductiva, que se pueden asociar a deficiencias de conocimientos operativos, organizativos, tecnológicos y técnicos (Kollias et al., 2013).

Contrario al caso de Grecia, en Colombia la respuesta frente a los altos niveles de violencia y criminalidad ha sido el aumento del gasto en justicia, defensa y seguridad para fortalecer las fuerzas armadas y el sistema judicial. De este modo, las políticas de un mayor gasto en seguridad han tenido resultados positivos en los últimos treinta ańos, pues han asumido implícitamente que un mayor gasto genera mayor eficiencia en el combate del crimen (González y Posada, 2001; 79). En la misma línea, Vargas y García (2008) argumentan que el gasto en defensa y seguridad en Colombia ha tenido una tendencia creciente a partir de la década de 1950 con oscilaciones debido a momentos coyunturales. Este crecimiento se ha hecho mayor a partir de la década de 1990, en el marco de la agudización del conflicto armado y el crecimiento del narcotráfico. El presupuesto destinado al sector se ha concentrado en el cubrimiento de gastos de funcionamiento, específicamente en gastos de personal, mientras que los gastos de inversión cuentan con un porcentaje menor de recursos.

En otro estudio, Loureiro y Carvalho-Junior (2007) analizaron la relación entre el gasto público en seguridad y crimen de Brasil. Los autores abordaron el problema desde la endogeneidad de las variables, para lo cual utilizaron valores rezagados de los ingresos del gobierno y el gasto en seguridad como variable instrumental. Los resultados encontrados sugieren que, al aumentar en uno por ciento en el gasto público en seguridad, la tasa de homicidios por cada 100000 habitantes se redujo en 0.21 por ciento, lo cual deja de manifiesto que el aumento en gasto público, al menos desde el análisis inferencial estadístico, coadyuvó en la reducción de la actividad delictiva.

En este punto de la discusión, la evidencia empírica sobre la relación e impacto del gasto público en seguridad y la actividad delictiva parece no tener un resultado único. Por lo tanto, estos resultados deben verse siempre con cautela, ya que las causas de la actividad delictiva son múltiples e incluso pueden llegar a estar interrelacionadas. Por ello, dependerá de cada análisis empírico señalar los alcances de cada medición estadística o econométrica, y de la metodología utilizada. En este sentido, desde la teoría se recomienda encontrar los múltiples determinantes de la actividad delictiva y aplicar la metodología que mejor se 
adapte a la naturaleza de la información disponible. Por ejemplo, Sachsida et al. (2010) analizan los determinantes de la actividad delictiva en Brasil. Se utilizó el método de datos de panel para información a nivel estatal sobre tasas de criminalidad. Una de las ventajas del enfoque de datos de panel según los autores es que permite incorporar la heterogeneidad no observada a nivel estatal. Los resultados econométricos también mostraron que el ingreso, la desigualdad, el desempleo y la urbanización están relacionados positivamente con la delincuencia. En este caso, los resultados mostraron que el gasto en seguridad pública es efectivo para disminuir la conducta delictiva. Asimismo, no se encontró evidencia de que la pobreza aumente el crimen. De hecho, los resultados indican una relación negativa entre pobreza y criminalidad. Finalmente, los resultados de las pruebas de causalidad de Granger mostraron que la desigualdad causa el crimen.

Por otra parte, Liu et al. (2013) analizaron el impacto que guardan algunas variables del mercado laboral con la actividad delictiva para el caso de México. Los resultados sugieren que salarios promedio más altos coadyuvan en menores tasas de criminalidad. Sin embargo, argumentan que las tasas de desempleo más bajas o el aumento del gasto en seguridad pública per cápita no logran una reducción en la actividad delictiva. También, sostienen que un mayor número de elementos de la policía federal, y mayores tasas de encarcelamiento tienen impactos significativos en la reducción del crimen.

En la misma línea, Orihuela y Lozano (2019) argumentan que el incremento en la tasa esperada de desempleo y la reducción de los salarios modifican los costos y beneficios que evalúan las personas antes de tomar la decisión sobre delinquir o no. Se encuentra que una disminución de los salarios tiene un efecto positivo en la oferta de crimen, debido a que disminuyen los beneficios legales frente a los ilegales; mientras que la tasa de desempleo actúa sobre los costos de delinquir, en el sentido de que una pérdida en las oportunidades en el mercado laboral disminuye los costos de oportunidad de las actividades ilegales. Los autores concluyen que las políticas destinadas a mejorar las perspectivas de empleo pueden ser una herramienta efectiva para combatir el crimen. Mejorar las oportunidades de empleo no es la única política para combatir el aumento de la delincuencia, Machin y Meghir (2004) enfatizan cómo las tasas de criminalidad en Reino Unido podrían verse influenciadas por la disminución de los salarios reales.

Raphael y Winter-Ebmer (2001) establecen que el desempleo es un determinante importante de las tasas de delitos contra la propiedad. Los autores encontraron evidencia de que el delito violento de robo con motivación económica es 
afectado positivamente por las tasas de desempleo. Este hallazgo, sin embargo, no se reproduce cuando se comparó el desempleo en los delitos de asesinato y violación.

Rodrigues (2018) establece una relación entre el gasto en seguridad, y la tasa de homicidios se evaluó a través de mínimos cuadrados de dos etapas. En primera instancia, se obtuvo un coeficiente significativo y negativo, lo que indica que un aumento de una unidad en el gasto per cápita conduce a una disminución en el número de homicidios por cada cien mil habitantes. Como señala la discusión de los resultados, estas estimaciones sugieren que un aumento de 1 por ciento en el gasto en seguridad conduce a una disminución de aproximadamente 0.4 por ciento en los homicidios en Brasil. En cuanto a los datos, la mayor preocupación es la existencia de errores en la medición de las variables en el tiempo, debido a un nuevo sistema de informes para el gasto público se implementó en 2002, por lo que es posible encontrar errores de medición en años anteriores, sesgando potencialmente los resultados.

Cortez y Grijalva (2021) reportan que, si bien no se observó un patrón claro en relación con el tamaño del impacto de la desigualdad, los resultados del análisis mostraron que, en general, el efecto es positivo, mayor desigualdad está asociada con mayor tasa de robo. La introducción del tamaño de municipios en el análisis permite inferir que la magnitud del impacto no es constante entre los municipios de diferente tamaño. Se encontró evidencia de que esta relación se torna negativa en municipios con poblaciones mayores de un millón de habitantes.

Por otro lado, Bourguignon (2000) señala cómo el crimen y la violencia son subproductos socialmente costosos de un desarrollo económico desigual. La teoría económica muestra cómo delitos contra la propiedad y la violencia asociada con la actividad ilegal son consecuencia de una desigualdad y pobreza excesivas. La evidencia limitada sugiere que un aumento en la pobreza relativa o la desigualdad de ingresos generalmente conducen a un aumento de la delincuencia. De manera similar, al aumentar el alcance de la pobreza, las recesiones importantes pueden inspirar un fuerte salto en el crimen. Es por esto que aumentos importantes de la desigualdad podrían generar pérdidas sociales de hasta 2 por ciento del PIB. Esta pérdida es aún mayor si se consideran solo las áreas urbanas, donde es probable que ocurra un delito.

En suma, los resultados empíricos sobre la relación entre el gasto público en seguridad y la actividad delictiva, dependerá en gran medida de la información disponible, de las variables que se tomen como explicativas de la actividad delictiva y de la metodología a utilizar. Por ello, el presente análisis se forja desde la 
teoría empírica aquí señalada y se advierte que los resultados deben ser tomados con cautela. La metodología se presenta a continuación.

\section{METODOLOGÍA}

En esta investigación se analiza la contribución del gasto público en materia de seguridad para mitigar los delitos en las entidades federativas de México a través de un micro panel balanceado, el cual se estructura con información de las 32 entidades mexicanas durante el periodo 2017-2019 para un total de 96 observaciones. Como consecuencia, se desarrolla la siguiente ecuación de delincuencia (1) en función del gasto en seguridad. Se consideran otras variables explicativas que, según la literatura, se relacionan a la variable de crimen.

$$
D_{i, t}=\alpha+\beta S_{i, t}+\gamma U_{i, t}+\delta W_{i, t}+\theta E_{i, t}+\varepsilon_{i, t}
$$

En donde $D$ representa la delincuencia, $S$ indica el gasto público en seguridad, $U$ es el desempleo, $W$ señala el salario, y $E$ simboliza la educación. Los parámetros $\gamma, \delta, \theta$ corresponden a los coeficientes a estimar, $\alpha$ es el intercepto. Mientras, $i$ y $t$ especifican la entidad y el año, respectivamente. Finalmente, $\varepsilon$ es el término de error estadístico y el coeficiente $\beta$ medirá el impacto del gasto de seguridad en la delincuencia, este último se espera que sea negativo.

Cabe mencionar que la estructura de la ecuación 1 cambiará dependiendo si se utilizan efectos aleatorios o fijos en las estimaciones. Para ello, se aplica la prueba Hausman (1978), cuya hipótesis nula sugiere el uso de efectos aleatorios; por lo tanto, en caso de rechazar dicha hipótesis, se asume el manejo de efectos fijos dentro de las estimaciones.

\section{Datos de las variables}

La variable explicada en la ecuación 1 es la delincuencia y se aborda en cuatro formas, como consecuencia se realizan cuatro estimaciones, las cuales son: delitos totales, delitos contra el patrimonio, delitos contra la libertad personal, y delitos contra la vida e integridad corporal (cuadro 1). La información delictiva se obtuvo a través del Secretariado Ejecutivo del Sistema Nacional de Seguridad Pública (SESNSP), que recopila los reportes estatales de las procuradurías generales de justicia, en donde se registran las denuncias realizadas ante el ministerio público. Dichas denuncias se utilizan para representar los delitos durante el análisis estadístico, esta medida se usa como una aproximación de los delitos reales puesto que existen casos donde no hay denuncia (Skogan, 1975). De acuerdo con 
CUADRO 1. Definición de las distintas clasificaciones de incidencia delictiva

\begin{tabular}{ll}
\hline \multicolumn{1}{c}{ Conceptos } & \multicolumn{1}{c}{ Definición } \\
\hline Delitos totales & Es la sumatoria conjunta de todas las distintas formas de incidencia delictiva. \\
Delitos contra el patrimonio & $\begin{array}{l}\text { Se contempla cualquier tipo de robo, además de fraude, extorsión, abuso de } \\
\text { confianza, daño a la propiedad, despojo, entre otros. }\end{array}$ \\
$\begin{array}{l}\text { Delitos contra la libertad } \\
\text { personal }\end{array}$ & $\begin{array}{l}\text { Se considera cualquier tipo de secuestro, también contiene los casos de tráfico } \\
\text { de menores y rapto. } \\
\text { Delitos contra la vida e } \\
\text { integridad corporal }\end{array}$ \\
\hline
\end{tabular}

Fuente: Elaboración propia con base en información del SESNSP.

González (2014), estas cifras de delincuencia se ponderan con la población de la entidad federativa y se calculan tasas de crecimiento; en otras palabras, se trabaja con la tasa de crecimiento anual de los delitos por cada mil habitantes para el periodo 2017-2019.

Asimismo, la variable independiente gasto público en seguridad se obtuvo por medio del SESNSP, que se encarga de publicar el presupuesto estatal del Fondo de Aportaciones para la Seguridad Pública (FASP).

Según el SESNSP (2021), el FASP es un fondo presupuestal previsto en la Ley de Coordinación Fiscal a través del cual se transfieren recursos a las entidades federativas para dar cumplimiento a estrategias nacionales en materia de seguridad pública. El FASP se orienta a diez programas con prioridad nacional:

1. Desarrollo de capacidades en las instituciones locales para el diseño de políticas públicas destinadas a la prevención social de la violencia y la delincuencia con participación ciudadana en temas de seguridad pública.

2. Desarrollo, profesionalización y certificación policial.

3. Tecnologías, infraestructura y equipamiento de apoyo a la operación policial.

4. Implementación y desarrollo del sistema de justicia penal y sistemas complementarios.

5. Fortalecimiento al sistema penitenciario nacional y de ejecución de medidas para adolescentes.

6. Desarrollo de las ciencias forenses en la investigación de hechos delictivos.

7. Sistema nacional de información para la seguridad pública.

8. Sistema nacional de atención de llamadas de emergencia y denuncias ciudadanas. 
9. Fortalecimiento de capacidades para la prevención y el combate a delitos de alto impacto.

10. Especialización de las instancias responsables de la búsqueda de personas.

Aquí mismo, es posible apreciar el monto destinado a todos programas de seguridad con prioridad nacional de cada entidad federativa, dicha cantidad se utiliza para el estudio, aunque en el ejercicio estadístico se implementa la tasa de crecimiento para el periodo 2017-2019. Antes de llevar a cabo los cálculos de las tasas, estas cifras monetarias se deflactan usando el índice nacional de precios al consumidor base junio 2018 del INEGI, con el objetivo de apreciar un cambio real de un periodo a otro. Por lo tanto, un incremento en el gasto de seguridad estará justificado siempre y cuando mitigue la incidencia delictiva; por consiguiente, y así como señalan Ivaschenko et al. (2012), se espera una relación inversa y significativa entre las tasas de seguridad y delincuencia. Cabe mencionar que, en caso de encontrar este hallazgo, no será posible argumentar eficiencia, puesto que los gobiernos logran combatir el crimen a costa de un mayor presupuesto.

La variable desempleo se representa por la tasa de desocupación, la cual se consiguió a través de la Encuesta Nacional de Ocupación y Empleo (eNOE). Dicha tasa se reporta cada tres meses, por lo que, se aplicó un promedio con las cifras de los cuatro trimestres del año para obtener una tasa anual. Esta relación es estudiada por Becker (1968), y corroborada por Carmichael y Ward (2000) y Wu y Wu (2012), en donde se estipula cómo una disminución de las fuentes de empleo incrementa la actividad delictiva, puesto que el deterioro de las oportunidades laborales reduce el costo de oportunidad del crimen. Por ello, se espera que el desempleo influya de forma positiva en la delincuencia.

Mientras, la variable salario se refleja a través del sueldo diario promedio de los trabajadores asegurados en el Instituto Mexicano del Seguro Social (IMSs), esta información se publica en la Secretaría de Trabajo y Previsión Social (STPS). Dichas cantidades monetarias se deflactan utilizando el índice nacional de precios al consumidor base junio 2018 del INEGi para el estudio estadístico. En consecuencia, estos datos proporcionan una aproximación del salario, pues solamente capturan el ingreso personal de quienes se encuentran en trabajos con esta prestación. Generalmente, se asume al salario como el costo de oportunidad por cometer un delito (Grogger, 1998); en este sentido, se espera una influencia inversa entre el salario y la delincuencia, dicha relación ha sido estudiada por Machin y Meghir (2004) y Braun (2019), quienes han encontrado una relación negativa. 
Finalmente, la educación se presenta como una fuente de mejores oportunidades, ya que genera capital humano y permite acceder a un ingreso mayor (Osborne y Higgins, 2015; Bennett, 2018), por lo que se espera una relación negativa con la delincuencia. Para representar la variable educación se utilizan los años promedio de escolaridad de las entidades mexicanas, esta medida es utilizada por Groot y van den Brink (2010), dicha información se obtuvo por medio de los reportes de indicadores educativos de la Secretaría de Educación Pública (SEP).

\section{Análisis descriptivo}

Un método útil para estudiar la relación entre la delincuencia y el gasto en seguridad es la gráfica de dispersión, la cual se construye por dos ejes: horizontal y vertical. En el horizontal se mide la tasa de crecimiento del gasto en seguridad, mientras la tasa de crecimiento de la delincuencia se refleja en el eje vertical, ambas descritas en la sección anterior.

De esta forma, se obtienen cuatro cuadrantes, lo que permite exhibir cuáles entidades incrementaron o disminuyeron el gasto en seguridad, para reducir o aumentar la cantidad de delitos. Por lo tanto, el cuadrante superior izquierdo mostrará los estados que disminuyeron su gasto e incrementaron el número de delitos, mientras los estados con aumentos en el gasto, pero con reducciones en la delincuencia se presentan en el cuadrante inferior derecho, en ambos espacios se percibe una relación negativa. No obstante, el cuadrante superior derecho contendrá a los estados que aumentaron el gasto en seguridad e incrementaron sus delitos, mientras las entidades con tasas de crecimiento negativas en el gasto de seguridad y delincuencia se encontrarán en el cuadrante inferior izquierdo.

La gráfica 1 muestra el gasto de seguridad y delincuencia para el ańo 2017, se observa cómo una mayoría considerable de estados redujeron el gasto destinado a seguridad, de los cuales Baja California Sur, Campeche, Guerrero, Morelos, Nayarit, Nuevo León, Sonora y Tamaulipas lograron mitigar la actividad criminal, al obtener tasas de crecimiento negativas en ambas variables, en donde sobresale Sonora, al ser el estado que más disminuyó la incidencia delictiva. Mientras, el resto de las entidades disminuyó el presupuesto en seguridad sin obtener un resultado favorable contra el crimen, pues estos aumentaron la actividad delictiva. Dentro de este rubro se destaca Sinaloa por ser el estado que más redujo el gasto en seguridad y obtuvo un leve incremento del crimen; por otro lado, Colima resultó la entidad más afectada por la delincuencia, pues este aumentó en un poco más del doble sus delitos respecto al año anterior. Sin embargo, únicamente Yucatán aumentó su presupuesto en seguridad y al mismo tiempo consiguió disminuir la tasa de delitos. 
GRÁFICA 1. Relación entre el gasto de seguridad y la delincuencia de las entidades de México, 2017

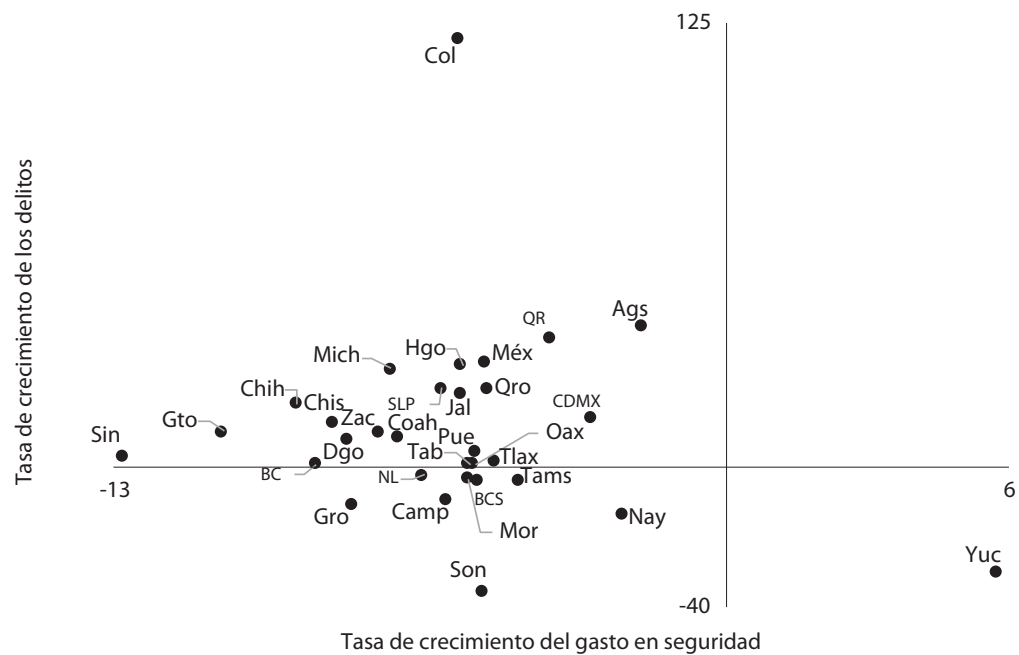

Fuente: Elaboración propia con base en datos del SESNSP.

En este sentido, si se toman como referencia los estados con las tasas más bajas de delitos (aquellos por debajo del eje de las abscisas de la gráfica 1), se puede analizar su proporción del gasto en prevención de delitos. Para ello, se muestra la gráfica 2, donde se aprecia que en el estado de Tamaulipas fue donde se destinó mayor proporción del gasto con 11.85 por ciento, seguido de Campeche con 7.94 por ciento, mientras que los estados restantes dedican cantidades por debajo de 5 por ciento del presupuesto.

Para estudiar la relación de 2018 se muestra la gráfica 3 que presenta una gran cantidad de entidades que incrementaron su presupuesto en una proporción similar, como, Aguascalientes, Campeche, Chihuahua, Hidalgo, Michoacán, Nayarit, Querétaro, Quintana Roo y Tabasco tuvieron un aumento en el gasto alrededor de 0.01 por ciento. De este grupo solamente Chihuahua y Tabasco consiguieron reducir el crimen, mientras el resto del grupo, junto con Morelos y Zacatecas, fueron incapaces de mitigar la delincuencia. En un sentido contrario al grupo anterior, aparece un grupo de estados con una disminución cerca de 9.5 por ciento, estos son Baja California, Baja California Sur, Durango, San Luis Potosí y Tamaulipas, en donde tres de estas entidades mostraron una reducción en sus 
GRÁFICA 2. Proporción del gasto destinado a la prevención en los estados con reducción de los delitos, 2017

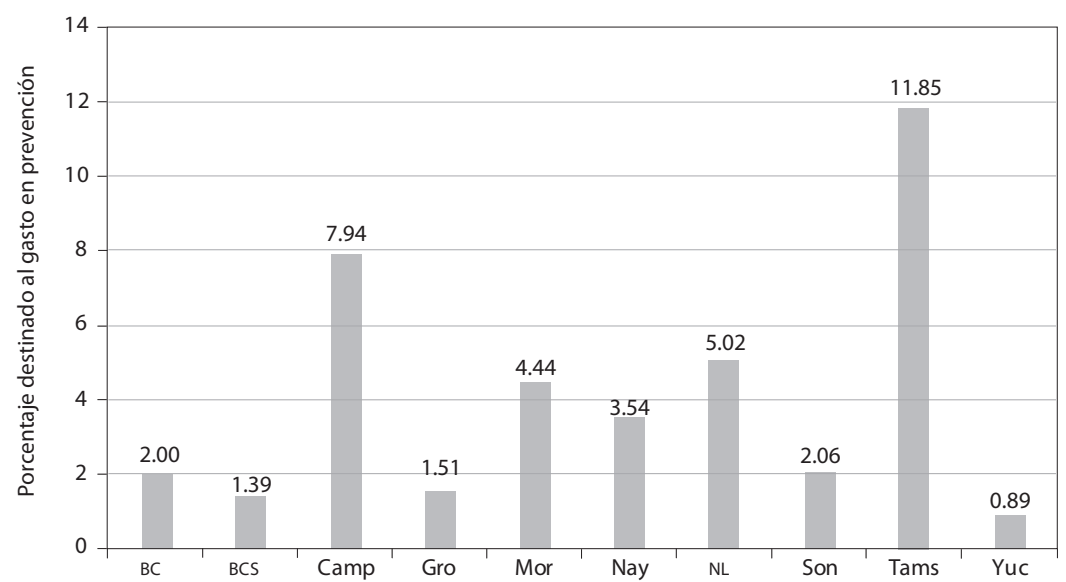

Fuente: Elaboración propia con base en datos del SESNSP.

GRÁFICA 3. Relación entre el gasto de seguridad y la delincuencia de las entidades de México, 2018

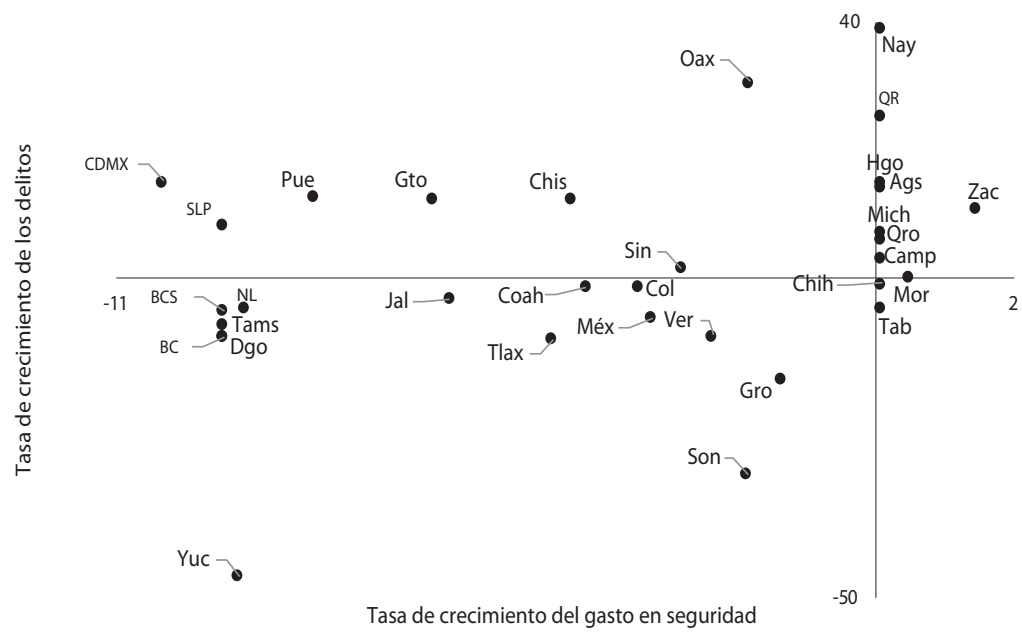

Fuente: Elaboración propia con base en datos del SESNSP. 
delitos, mientras San Luis Potosí fue el único del grupo en incrementar actividad delictiva. Además de San Luis, aparecen Chiapas, Ciudad de México, Guanajuato, Oaxaca, Puebla y Sinaloa dentro del mismo cuadrante, donde se reduce el gasto de seguridad y se incrementa la incidencia criminal. En general, durante este año, la mayoría de las entidades recibieron un presupuesto menor en seguridad, quizá por el hecho de ser un año electoral y consiguieron minorar la delincuencia. En una observación individual, sobresale Yucatán como el estado que más redujo la iniciativa delictiva con una disminución de su gasto en seguridad, en oposición a Nayarit, que tiene la tasa más alta de delitos.

Ahora bien, si se toma como referencia a los estados con las tasas de crecimiento más bajas de delitos, se puede analizar su proporción del gasto en prevención de delitos. Para ello, se muestra la gráfica 4. Resalta el caso de Yucatán, que de acuerdo con las gráficas 1 y 2 fue el estado con la segunda tasa de crecimiento de delitos más baja y al mismo tiempo aumentó su gasto en seguridad, pero solo el 0.89 por ciento lo destinó a la prevención de delitos. Para 2018, Yucatán siguió manteniendo una tasa de crecimiento de delitos baja, pero con una reducción del gasto en seguridad y con un incremento en la proporción del gasto en la prevención de delitos que pasó a 21.14 por ciento.

GRÁFICA 4. Proporción del gasto destinado a la prevención en los estados con reducción de delitos, 2018

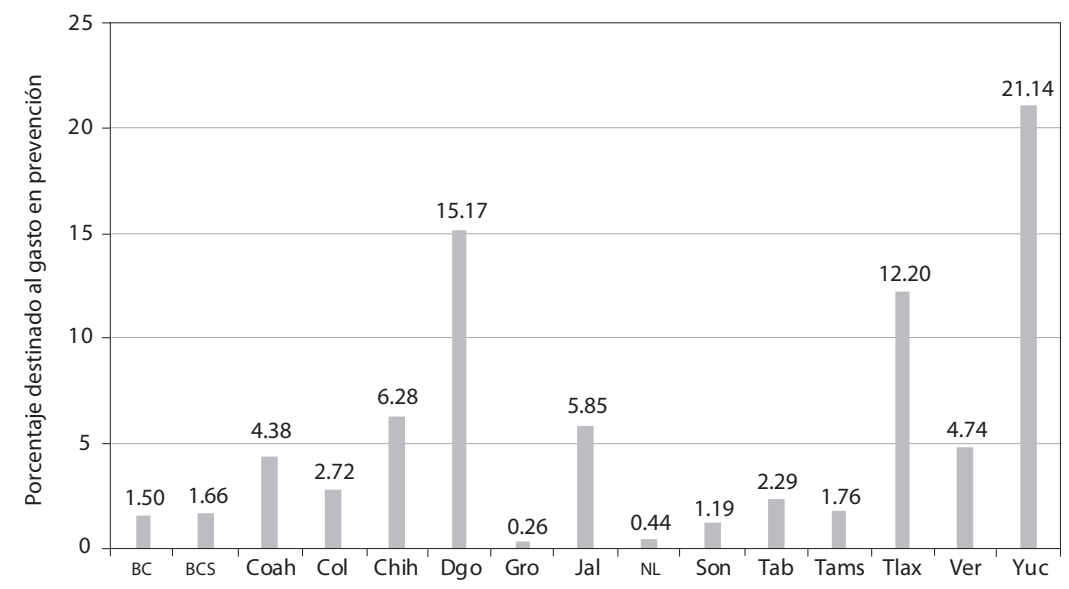

Fuente: Elaboración propia con base en datos del SESNSP. 
GRÁFICA 5. Relación entre el gasto de seguridad y la delincuencia de las entidades de México, 2019

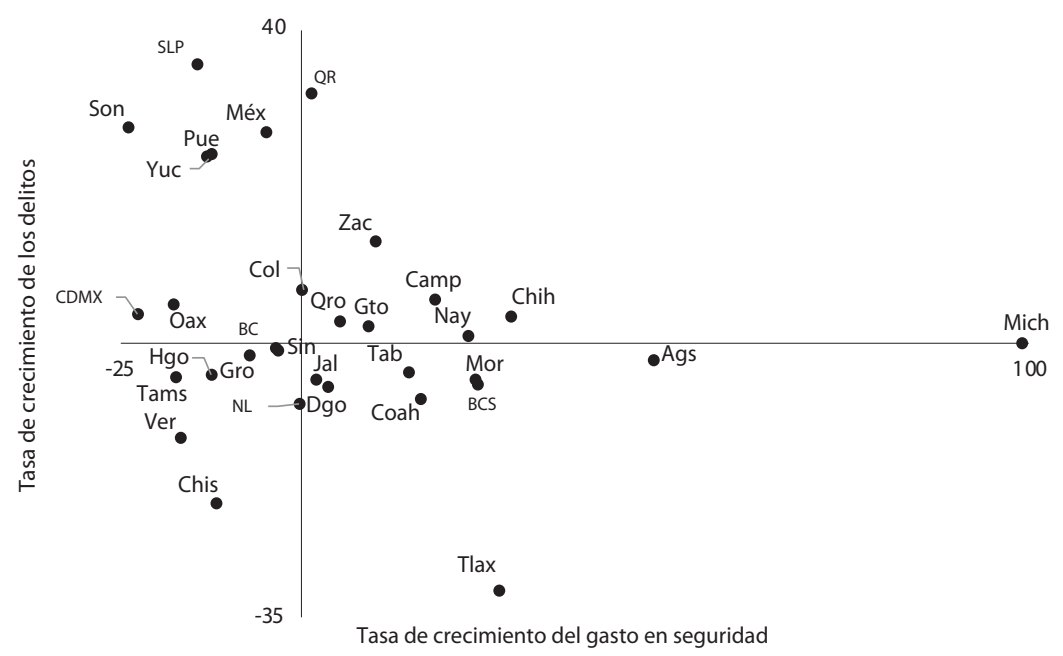

Fuente: Elaboración propia con base en datos del SESNSP.

En la gráfica 5 se muestra la relación entre seguridad y delincuencia para 2019. En este ańo, se aprecia a Michoacán como la entidad con la mayor tasa de crecimiento del gasto, y a Sonora como el estado que más redujo el presupuesto en seguridad. Respecto a la delincuencia, las cifras extremas corresponden a Tlaxcala y San Luis Potosí, al ser las entidades que más disminuyeron el presupuesto y donde aumentaron los delitos, respectivamente.

Se observa a la Ciudad de México, Estado de México, Puebla, Oaxaca, San Luis Potosí, Sonora y Yucatán como los estados donde se disminuyó el gasto y se incrementaron los delitos, mientras que las entidades donde se aumentó el gasto en seguridad y disminuyó la delincuencia son Aguascalientes, Baja California Sur, Coahuila, Durango, Jalisco, Michoacán, Morelos, Tabasco y Tlaxcala. Por otro lado, Campeche, Chihuahua, Colima, Guanajuato, Nayarit, Querétaro, Quintana Roo y Zacatecas incrementaron su presupuesto en seguridad sin reducir la actividad criminal; en sentido contrario, entidades como Baja California, Chiapas, Guerrero, Hidalgo, Sinaloa, Tamaulipas y Veracruz consiguieron disminuir la actividad criminal sin requerir un aumento del gasto en seguridad. 
GRÁFICA 6. Proporción del gasto destinado a la prevención en los estados con reducción de los delitos, 2019

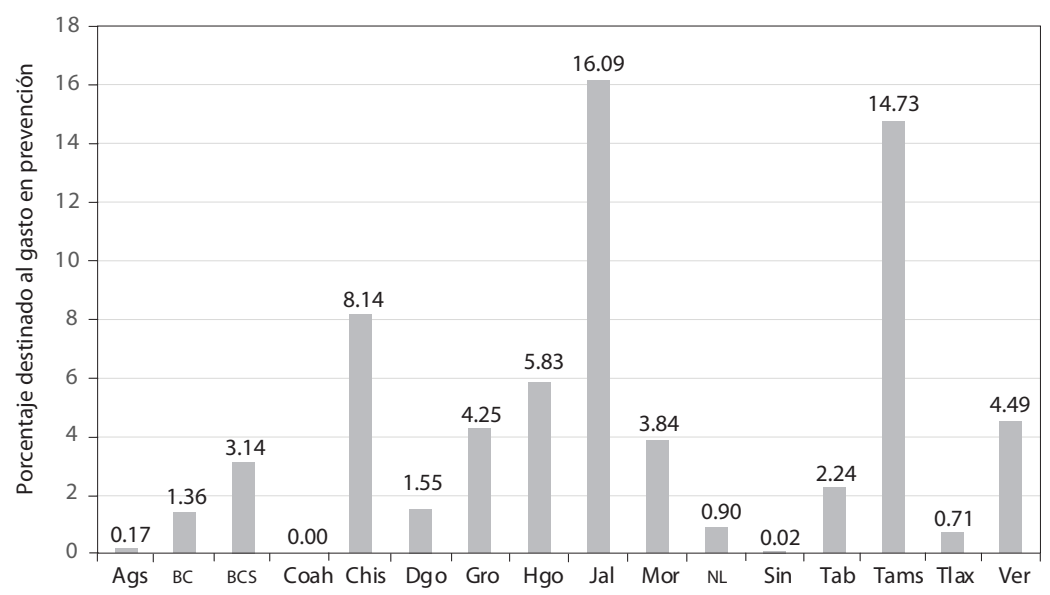

Fuente: Elaboración propia con base en datos del SESNSP.

En la gráfica 6 se presenta lo referente al gasto destinado a prevención de delitos en 2019. Jalisco es el que muestra una mayor participación con 16.09 por ciento, seguido de Tamaulipas con 14.73 por ciento y Chiapas con 8.14 por ciento. El resto de los estados tuvieron participaciones por debajo de 6 por ciento de su presupuesto asignado.

$\mathrm{Al}$ analizar las gráficas 2, 4 y 6, no se puede establecer una conclusión sólida para afirmar que un aumento en la prevención de delitos, coadyuva a reducir la tasa de crecimiento de delitos. Por ejemplo, el caso de Yucatán, donde se tenía una tasa de crecimiento de delitos baja y al mismo tiempo un incremento en el gasto de prevención, para 2019 el resultado fue diferente.

Dados estos resultados, donde la mayoría de los estados destinan una baja proporción del presupuesto en seguridad en la prevención de delitos resalta el estudio de Fuentes y Sánchez (2017), quienes sostienen que en México poco se ha hecho en materia de prevención, y que los mecanismos para mitigar la actividad delictiva se sujetan a la realidad que enfrenta cada estado.

Asimismo, al sintetizar el análisis de las gráficas 1, 2 y 3, que permite visualizar el efecto entre la delincuencia y el gasto en seguridad, esta relación no ha sido constante entre los años analizados. Si bien, en 2017 y 2019 se exhibe una ligera 
relación negativa entre las tasas de los delitos y el gasto de las entidades, estos dos años presentan situaciones distintas; por un lado, en 2017 hubo un gran número de estados que disminuyeron su presupuesto en seguridad y, a la vez, gran parte de estos obtuvieron un incremento en el crimen, a diferencia del año 2019, cuando los estados presentan situaciones heterogéneas y no hay un grupo con mayoría dominante, es decir, la cantidad de entidades es muy similar en cada cuadrante. Por otra parte, para 2018 se aprecia una leve tendencia positiva, aunado a una estremecedora cantidad de estados que disminuyeron los delitos sin necesidad de aumentar el gasto.

Cabe mencionar que estos hallazgos deben ser considerados con cautela, puesto que este tipo de ejercicio sólo examina la relación entre dos variables, pero ignora los efectos de otros fenómenos. Aunque se cuenta con vasta literatura, es importante continuar con el estudio de las trayectorias resultantes por parte del gasto de seguridad y la delincuencia en México, pues esto permitirá que los tomadores de decisiones en materia de política pública logren implementar políticas que coadyuven a mitigar la actividad delictiva.

\section{RESULTADOS}

En esta sección se presentan los resultados de la contribución del gasto público en seguridad sobre: los delitos totales; delitos contra el patrimonio; delitos contra la libertad, y delitos contra la vida e integridad corporal (cuadro 2). Cabe señalar que previamente se aplicó la prueba de Hausman para contrastar el tipo de efecto en las estimaciones de datos de un micro panel, su hipótesis especifica el uso de efectos aleatorios, los resultados de dicha prueba no permiten rechazar la hipótesis nula al obtener probabilidades mayores a 5 por ciento, por consiguiente, cada estimación se realiza con efectos aleatorios.

En el cuadro 2, la primera estimación de delincuencia corresponde a la variable de delitos totales, esta muestra una relación negativa y significativa con el gasto público en seguridad, en donde un aumento en una unidad porcentual de la tasa del gasto en seguridad disminuye el crecimiento de los delitos en un 0.21 por ciento. A su vez, el salario es otro fenómeno estadísticamente relevante en el crimen, pues un incremento en una unidad del salario aumenta en casi 0.05 por ciento la actividad delictiva. Mientras, el desempleo y la educación no mostraron significancia estadística, aunque ambas reportan el signo esperado.

Los resultados de la delincuencia contra el patrimonio son muy similares a los obtenidos con los delitos totales (cuadro 2), al encontrar cómo el gasto en seguridad también influye de forma considerable en los delitos patrimoniales, pues un 
CUADRO 2. Estimaciones de la delincuencia y gasto en seguridad en las entidades de México, 2017-2019

\begin{tabular}{|c|c|c|c|c|}
\hline \multirow{2}{*}{$\begin{array}{c}\text { Variables } \\
\text { independientes }\end{array}$} & \multicolumn{4}{|c|}{ Variable dependiente } \\
\hline & Delitos & $\begin{array}{l}\text { Delitos contra } \\
\text { el patrimonio }\end{array}$ & $\begin{array}{l}\text { Delitos contra la } \\
\text { libertad personal }\end{array}$ & $\begin{array}{c}\text { Delitos contra } \\
\text { la vida y la } \\
\text { integridad } \\
\text { corporal }\end{array}$ \\
\hline Constante & $\begin{array}{r}19.9725 \\
(18.4519)\end{array}$ & $\begin{array}{r}25.0311 \\
(15.3409)\end{array}$ & $\begin{array}{r}24.4235 \\
(70.7916)\end{array}$ & $\begin{array}{r}15.5379 \\
(11.1572)\end{array}$ \\
\hline $\begin{array}{l}\text { Gasto en } \\
\text { seguridad }\end{array}$ & $\begin{array}{l}-0.2153^{* * * *} \\
(0.0805)\end{array}$ & $\begin{array}{l}-0.2529 * * * \\
(0.0880)\end{array}$ & $\begin{array}{r}-0.3322 \\
(0.3291)\end{array}$ & $\begin{array}{r}-0.1202 \\
(0.0992)\end{array}$ \\
\hline Desempleo & $\begin{array}{r}0.7620 \\
(1.8418)\end{array}$ & $\begin{array}{r}0.5163 \\
(0.0190)\end{array}$ & $\begin{array}{r}6.3184 \\
(4.8276)\end{array}$ & $\begin{array}{r}2.1274 \\
(1.3237)\end{array}$ \\
\hline Salario & $\begin{array}{r}-0.0480 \\
(0.0164)\end{array}$ & $\begin{array}{l}-0.0604^{* * *} \\
(0.0190)\end{array}$ & $\begin{array}{l}-0.8122 * \\
(0.4758)\end{array}$ & $\begin{array}{l}-0.0691 \\
(0.0432)\end{array}$ \\
\hline Educación & $\begin{array}{r}-0.1975 \\
(2.8611)\end{array}$ & $\begin{array}{r}-0.5383 \\
(2.5973)\end{array}$ & $\begin{array}{r}28.3601 \\
(18.1660)\end{array}$ & $\begin{array}{r}0.4927 \\
(1.6344)\end{array}$ \\
\hline $\begin{array}{l}\text { Número de } \\
\text { observaciones }\end{array}$ & 96 & 96 & 96 & 96 \\
\hline
\end{tabular}

Fuente: Elaboración propia. Nota: Se consideran 32 unidades de sección cruzada y 3 unidades de tiempo. ${ }^{* *}{ }^{*}{ }^{*}$ representa significancia estadística al nivel 0.01 y 0.1 , respectivamente. Los valores entre paréntesis corresponden a los errores estándar.

aumento de 1 por ciento en la tasa del gasto gubernamental reduce aproximadamente 0.25 por ciento el crecimiento de los delitos contra el patrimonio. De igual forma, el salario se manifiesta de manera negativa contra este tipo de delincuencia, dado que un incremento en una unidad del salario promedio real reduciría en 0.06 por ciento el crecimiento de actos ilícitos contra el patrimonio.

Respecto a la estimación de los delitos contra libertad personal, el principal resultado se obtiene con la variable salario al ser estadísticamente significativo y presentar un coeficiente negativo, por lo tanto, un aumento del salario real en una unidad monetaria disminuiría en 0.81 por ciento la tasa de crecimiento del crimen contra la libertad personal. Entretanto, la relación entre el gasto de seguridad y la delincuencia contra la libertad de las personas es inversa; sin embargo, esta no resultó relevante en términos estadísticos. De la misma manera, las variables independientes desempleo y educación presentan una relación acorde a lo esperado con los delitos patrimoniales, pero carecen de significancia estadística. 
La estimación de la variable delincuencia contra la vida y la integridad corporal muestra una relación negativa con el gasto público en seguridad; no obstante, dicha relación no es estadísticamente significativa (cuadro 2). Un resultado análogo se presenta con las otras variables de control, las cuales reportan coeficientes con el signo esperado, pero no cuentan presencia relevante en términos estadísticos para los delitos que atentan contra la vida, al no reportar significancia en ninguno de los niveles convencionales.

En resumen, los resultados del cuadro 2 manifiestan una contribución del gasto gubernamental de seguridad para combatir la delincuencia, pero dicho impacto es efectivo en los delitos totales y contra el patrimonio, y no así en los crímenes relacionados con la libertad, la vida e integridad de las personas, pues estos últimos no cumplen con las cláusulas estadísticas de significancia, a pesar de ostentar una relación de acuerdo con lo esperado. Por otra parte, el salario se presenta como un elemento que coadyuva en la disminución de la delincuencia, al influir de forma negativa y práctica en los crímenes totales, contra el patrimonio y la libertad personal. Mientras, desempleo y educación no resultaron ser componentes capaces de afectar el nivel de delitos en México durante el periodo estudiado, si bien ambas variables exhiben coeficientes con el signo esperado, estos omiten el rigor estadístico al incumplir con el mínimo nivel de significancia comúnmente aceptado.

\section{CONCLUSIONES}

Analizar y determinar los factores que inciden en la mitigación de la actividad delictiva es fundamental para la toma de decisiones de política pública de los gobiernos. No obstante, al existir múltiples factores que se expresan como causas de la actividad delictiva, resulta complejo llegar a una conclusión unánime respecto de cuál debería ser la mejor estrategia para mitigar esta problemática. Se pueden emplear estrategias de corto y largo plazo, pero la única constante en el análisis es el gasto público que destinan los gobiernos en el combate a la inseguridad. El problema es que, desde la teoría empírica, no queda claro si un mayor gasto en seguridad efectivamente incide en la mitigación de la actividad delictiva o, tal como sugieren Astudillo y Porras (2021), si destinar el gasto público a la implementación de estrategias e inversiones productivas que inciden directamente en el ámbito socioeconómico, indirectamente se mitiga la actividad delictiva. En este sentido, el elemento central para destinar un mayor presupuesto a seguridad pasa por determinar el impacto que este tiene en la disminución de la actividad delictiva.

En México, se reconoce que la inseguridad como consecuencia de la actividad delictiva es el principal problema social que enfrenta la sociedad. Es por ello que 
los gobiernos estatales en algunos casos incrementaron el gasto público; sin embargo, otros estados destinan un menor presupuesto en el ámbito de seguridad. Como resultado, la mayoría de los estados lograron disminuir la incidencia delictiva, sobre todo en 2017 y 2019. No obstante, en otros casos el resultado no fue el mismo. Lo anterior muestra que, a pesar de seguir una misma estrategia, los resultados no son homogéneos, debido principalmente a los múltiples factores que inciden en las causas de la actividad delictiva.

En este sentido, un análisis empírico más integral resulta más apropiado, donde, además de incorporar la relación entre el gasto público en seguridad y la actividad delictiva, es necesaria la inclusión de otras variables. Por ello los múltiples modelos econométricos sugieren al menos una relación inversa entre el gasto público en seguridad y la actividad delictiva. Esto sugiere que emplear un mayor presupuesto en seguridad coadyuva en la mitigación de los delitos. Cabe señalar que estos resultados deben tomarse con total cautela, ya que dependiendo de la metodología empleada e información disponible los resultados pueden arrojar otras conclusiones, muestra de ello es que, para las diferentes variables dependientes utilizadas en cada modelo, la significancia estadística no se mantuvo.

Con relación al efecto entre la actividad delictiva y el desempleo, no se encontró significancia estadística. Caso contrario con el salario, pues de acuerdo con los resultados un mayor salario produce una disminución en la actividad delictiva. En este sentido, la estrategia del gobierno federal de aumentar el salario mínimo puede representar una alternativa para mitigar la actividad delictiva. Respecto a la educación, no se encontró evidencia estadísticamente significativa para establecer que una mayor educación pudiera representar una menor incidencia delictiva.

En suma, se puede establecer que un mayor gasto público en seguridad es necesario para mitigar la actividad delictiva. Asimismo, queda para futuras investigaciones analizar cuál es la mejor alternativa para destinar el gasto público en seguridad. Es decir, qué resulta en una mayor eficiencia: si el gasto destinado a la prevención del delito, el gasto destinado al combate al crimen o el gasto destinado a la procuración de justicia. $G \cong \Xi$

\section{REFERENCIAS}

Astudillo, M. y R. Porras (2021), "Modelo de contabilidad social para México basado en cuentas por sectores institucionales", Gestión y Politica Pública, XXX(1), pp. 61-99, Dor: doi.org/10.29265/gypp.v30i1.810.

Becker, G. (1968), "Crime and Punishment: An Economic Approach", The Economic Dimensions of Crime, 13(68), pp. 13-68, Dor: doi.org/10.1007/978-1-349-62853-7_2. 
Bennett, P. (2018), “The Heterogeneous Effects of Education on Crime: Evidence from Danish Administrative Twin Data", Labour Economics, 52, 160-177, DoI: doi.org/ 10.1016/j.labeco.2018.02.002.

Bourguignon, F. (2000), "Crime, Violence and Inequitable Development", en Annual World Bank Conference on Development Economics, Washington, D.C., The World Bank, pp. 199-220.

Braun, C. (2019), "Crime and the Minimum Wage", Review of Economic Dynamics, 32, pp. 122-152, DoI: doi.org/10.1016/j.red.2019.02.002.

Carmichael, F. y R. Ward (2000), Youth Unemployment and Crime in the English Regions and Wales, Applied Economics, 32(5), pp. 559-571, Dor: doi.org/10.1080/000368400322462. CIEP (Centro de Investigación Económica y Presupuestaria) (2019), Gasto en seguridad: Una mirada a la ruta de los recursos, Ciudad de México, Observatorio Nacional Ciudadano.

Cortez, W.W. y A.E. Grijalva Eternod (2021), "Pobreza, desigualdad y tamaño de municipio como factores explicativos del robo en México", Gestión y Política Pública, XXX(1), pp. 127-161.

Daniele, V. y U. Marani (2008), "Organized Crime and Foreign Direct Investment: The Italian Case”, cesifo Working Paper Series 2416, cesifo.

Foglia, M. (2017), "La participación Argentina en la agenda de la cooperación de seguridad y lucha contra la criminalidad organizada de la UNASUR", Revista de Estudios en Seguridad Internacional, 3(1), pp. 61-79.

Fuentes, C.M. y O.A. Sánchez (2017), "La distribución espacial del robo a transeúntes y el contexto socioeconómico en tres delegaciones de la Ciudad de México: Elementos para una política de seguridad pública”, Gestión y Política Pública, XXVI(2), pp. 417-451.

González, F. y C. Posada (2001), "Criminalidad, violencia y gasto público en justicia, defensa y seguridad en Colombia”, Revista de Economía Institucional, 3(4), pp. 78-102.

González, S. (2014), "Criminalidad y crecimiento económico regional en México", Frontera Norte, 26(51), pp. 75-111.

Grogger, J. (1998), "Market Wages and Youth Crime”, Journal of Labor Economics, 16(4), pp. 756-791, Dor: doi.org/10.3386/w5983.

Groot, W. y H. van den Brink (2010), "The Effects of Education on Crime: Applied Economics”, 42(3), pp. 279-289, DoI: doi.org/10.1080/00036840701604412.

Hausman, J.A. (1978), Specification Tests in Econometrics, Econometrica, 46(6), pp. 1251-1271. INEGI (Instituto Nacional de Estadística y Geografía) (2019), Encuesta Nacional de Victimización y Percepción sobre Seguridad Publica, principales resultados, disponible en: https://www.inegi.org.mx/contenidos/programas/envipe/2019/doc/envipe2019_presentacion_nacional.pdf 
Ivaschenko, O., A. Nivorozhkin y E. Nivorozhkin (2012), "The Role of Economic Crisis and Social Spending in Explaining Crime in Russia", Eastern European Economics, 50(4), pp. 21-41, Dor: doi.org/10.2753/eee0012-8775500402.

Jaitman, L. y P. Keefer (2017), “¡Por qué es importante la estimación de los costos del crimen? Una agenda de investigación para apoyar las políticas de prevención del delito en la región", en L. Jaitman (ed.), Los costos del crimen y de la violencia, nueva evidencia y hallazgos en América Latina y el Caribe, Washington, D.C., Banco Interamericano de Desarrollo, pp. 1-17.

Kollias, C., N. Mylonidis y S.M. Paleologou (2013), "Crime and the Effectiveness of Public Order Spending in Greece: Policy Implications of Some Persistent Findings", Journal of Policy Modeling, 35(1), pp. 121-133, DoI: doi.org/10.1016/j.jpolmod.2012.02.004.

Liu, Y., T.M. Fullerton Jr. y N.J. Ashby (2013), “Assessing the Impacts of Labor Market and Deterrence Variables on Crime Rates in Mexico", Contemporary Economic Policy, 31(4), pp. 669-690, DoI: doi.org/10.1111/j.1465-7287.2012. 00339.x.

Loureiro, A.O. y J.R. Carvalho-Junior (2007), O impacto dos gastos públicos sobre a criminalidade no Brasil, disponible en: http://www.anpec.org.br/encontro2007/artigos/ A07A163.pdf [fecha de consulta:].

Martínez, G. y A.L. Morones (2019), “Análisis de los determinantes de la percepción de la corrupción como problema social en México", Revista Legislativa de Estudios Sociales y de Opinión Pública, 12(25), pp. 163-184.

Machin, S. y C. Meghir (2004), "Crime and Economic Incentives”, The Journal of Human Resources, 39(4), pp. 958-979, Dor: doi.org/10.2307/3559034.

Orihuela, D.V. y L. Lozano (2019), "El aumento del crimen y la disminución de la participación laboral en el ingreso en México", Apuntes del CENES, 38(68), pp. 49-78.

Osborne, D. y G. Higgins (2015), "Education and Crime", The Encyclopedia of Crime and Punishment, pp. 1-5, DoI: doi.org/10.1002/9781118519639.wbecpx183.

Raphael, S. y R. Winter-Ebmer (2001), "Identifying the Effect of Unemployment on Crime", The Journal of Law and Economics, 44(1), pp. 259-283, DOI: doi.org/10.1086/ 320275.

Rodrigues, C. (2018), Crime and Government Expenditure in Brazil: Estimating the Impact of Government Security Spending on Homicide Rates, Washington, D.C., Banco Interamericano de Desarrollo, DoI: dx.doi.org/10.18235/0001365.

Sachsida, A., M.J. Cardoso, P. Loureiro y M. Sarmiento (2010), "Inequality and Criminality Revisited: Further Evidence from Brazil”, Empirical Economics, 39(1), pp. 93-109, DOI: doi.org/10.1007/s00181-009-0296-4.

SESNSP (Secretariado Ejecutivo del Sistema Nacional de Seguridad Pública) (2021), Fondo 
de Aportaciones para la Seguridad Pública (FASP), disponible en: https://www.gob.mx/ sesnsp/es/\#2696 [fecha de consulta: 10 de julio de 2021].

Skogan, W. (1975), "Measurement Problems in Official and Survey Crime Rates", Journal of Criminal Justice, 3(1), pp. 17-31, Dor: doi.org/10.1016/0047-2352(75)90096-3.

Soria, R. (2018), "Una estimación del costo de la inseguridad y la delincuencia en México: Análisis comparativo a nivel de las entidades federativas", Gestión y Política Pública, XXVII(1), pp. 111-147.

Sumano, J.A. (2020), "Prevención del delito en la frontera norte a través de la administración pública deliberativa", Gestión y Politica Pública, XXIX(1), pp. 67-96, DoI: doi. org/10.29265/gypp.v29i1.657.

Vargas, A. y V. García (2008), "Seguridad ciudadana y gasto público: Reflexiones sobre el caso colombiano", América Latina Hoy, 50, pp. 37-51, Dor: doi.org/10.14201/alh.1339.

Vilalta Perdomo, C. y G. Fondevila (2018), "La victimización de las empresas en México: Conceptos, teorías y mediciones”, Gestión y Política Pública, XXVII(2), pp. 501-540.

Wu, D. y Z. Wu (2012), "Crime, Inequality and Unemployment in England and Wales: Applied Economics, 44(29), pp. 3765-3775, Dor: doi.org/10.1080/00036846.2011.581217.

Zúniga, L. (2016), "El concepto de criminalidad organizada transnacional: Problemas y propuestas", Nuevo Foro Penal, 86, pp. 62-114, DoI: doi.org/10.17230/nfp.12.86.2.

Carlos Heriberto Flores Orona cursa actualmente una maestría en Administración Pública. Es doctor en Ciencias Económicas por la Universidad Autónoma de Baja California en Tijuana, México (2017). También es maestro en Economía Aplicada por El Colegio de la Frontera Norte en Tijuana, México (2014), además de que cuenta con una licenciatura en Economía por la Universidad Autónoma de Ciudad Juárez, México (2011). Actualmente ejerce como profesor de cátedra en el Instituto Tecnológico y de Estudios Superiores de Monterrey (ITESM) campus Chihuahua. Imparte asignaturas en la Escuela de Negocios y Gobierno de Econometría Financiera I, Econometría Financiera II y Emprendimiento. Sus líneas de investigación son seguridad, migración, crecimiento económico y series de tiempo.

Rafael Eduardo Saavedra Leyva es doctor en Ciencias Económicas por la Universidad Autónoma de Baja California en Tijuana, México (2017). También ostenta una maestría en Ciencias Económicas por la Universidad Autónoma de Baja California en Tijuana, México (2014), además de una licenciatura de Ingeniería Industrial por el Instituto Tecnológico de Culiacán en Culiacán, México (2011). Actualmente ejerce como profesor de tiempo completo de la Facultad de Ciencias Químicas e Ingeniería en la Uni- 
versidad Autónoma de Baja California en Tijuana, México. Imparte asignaturas de la línea económico-administrativa para las carreras de Ingeniería Industrial e Ingeniería Química. Su línea de investigación es desarrollo empresarial y emprendimiento. Es candidato a investigador nacional desde 2020.

Gilberto Martínez Sidón es licenciado en Economía por la Universidad Autónoma de Baja California Sur (2007-2011). Maestro en Economía Aplicada por el Colegio de la Frontera Norte (2012-2014). Doctor en Ciencias Económicas por la Universidad Autónoma de Baja California (2014-2017). Profesor de asignatura en la Universidad Autónoma de Baja California en el periodo 2015-2017. Profesor de asignatura en el Tecnológico Nacional de México, Instituto Tecnológico de la Paz, Baja California Sur, 2018. Profesor-investigador del Departamento Académico de Economía de la Universidad Autónoma de Baja California Sur en 2018. Pertenece al Sistema Nacional de Investigadores en el nivel candidato desde 2020. Su área de especialización de investigación es: economía regional y sectorial. Sus líneas de investigación son el crecimiento y desarrollo económicos. 
